\title{
THE BIPARTITE-SPLITTANCE OF A BIPARTITE GRAPH $^{1}$
}

\author{
JiAn-HuA Yin AND Jing-Xin GuAN \\ Department of Mathematics \\ College of Information Science and Technology \\ Hainan University, Haikou 570228, P.R. China \\ e-mail: yinjh@hainu.edu.cn
}

\begin{abstract}
A bipartite-split graph is a bipartite graph whose vertex set can be partitioned into a complete bipartite set and an independent set. The bipartitesplittance of an arbitrary bipartite graph is the minimum number of edges to be added or removed in order to produce a bipartite-split graph. In this paper, we show that the bipartite-splittance of a bipartite graph depends only on the degree sequence pair of the bipartite graph, and an easily computable formula for it is derived. As a corollary, a simple characterization of the degree sequence pair of bipartite-split graphs is also given.
\end{abstract}

Keywords: degree sequence pair, bipartite-split graph, bipartite-splittance.

2010 Mathematics Subject Classification: 05C07.

\section{REFERENCES}

[1] S. Földes and P.L. Hammer, Split graphs, in: Proc. 8th Sout-Eastern Conf. on Combinatorics, Graph Theory and Computing, F. Hoffman et al. (Ed(s)), (Baton Rouge, Lousiana State University, 1977) 311-315.

[2] D. Gale, A theorem on flows in networks, Pac. J. Math. 7 (1957) 1073-1082. doi:10.2140/pjm.1957.7.1073

[3] P.L. Hammer and B. Simeone, The splittance of a graph, Combinatorica 1 (1981) 275-284. doi:10.1007/BF02579333

\footnotetext{
${ }^{1}$ Supported by National Natural Science Foundation of China (No. 11561017) and Natural Science Foundation of Hainan Province (No. 2016CXTD004).
} 
[4] H.J. Ryser, Combinatorial properties of matrices of zeros and ones, Canad. J. Math. 9 (1957) 371-377.

doi:10.4153/CJM-1957-044-3

Received 12 May 2016

Revised 18 April 2017

Accepted 18 April 2017 